\title{
Marcadores discursivos: apréndelos y tradúcelos
}

\author{
SHIMA SALAMEH JIMÉNEZ \\ Universitat de València \\ shima.salameh@uv.es
}

\begin{abstract}
Resumen: La enseñanza de los marcadores discursivos siempre presenta dificultades en la clase de E/LE: aprender un significado procedimental cuesta más que aprender uno conceptual. Traducirlos tampoco es fácil: no todos los marcadores discursivos tienen un equivalente directo en otras lenguas (Rossari 1990). Proponemos una serie de actividades para el aula del traductor alemán-español, dentro de un grupo heterogéneo de alumnos con un nivel de español medio-alto. Las actividades propuestas giran en torno a los distintos niveles lingüísticos con los que se caracteriza a los marcadores (prosódico, morfosintáctico, semántico y pragmático-discursivo). Con ellas, aprenderán a detectar marcadores discursivos en varios medios (escrito y oral) para traducirlos adecuadamente al español. Asimismo, se familiarizarán con nuevas actividades y herramientas de trabajo (Diccionario de Partículas Discursivas del Español-DPDE, Briz, Pons y Portolés 2008-; Corpus de Conversaciones Coloquiales Val.Es.Co. -Briz y grupo Val.Es.Co. 2002-; Corpus Val.Es.Co. 2.0.-Cabedo y Pons 2013-).
\end{abstract}

Palabras clave: marcadores discursivos, traducción, español coloquial, enfoque comunicativo, Val.Es.Co., DPDE.

\section{Discourse markers: learn and translate them!}

Abstract: Learning and studying discourse markers is quite complex: on the one hand, students always present problems at learning their procedural meanings (because this task is harder than learning conceptual meanings). On the other hand, to translate their meaning is also complicated: not all the languages share equivalent discourse markers (Rossari 1990). This didactic proposal aims at offering various resources for the translator who also studies Spanish. These activities address different discourse markers (formal and colloquial) from different linguistic levels (phonologic, morphosyntactic, semantic and pragmatic-discursive). Such activities will allow translators for learning the meaning of discourse markers in both written and conversational contexts and, in turn, knowing new tools to translate them with less difficulties in a systematic way (Diccionario de Particulas Discursivas del Español -DPDE, Briz, Pons y Portolés 2008-; Corpus de Conversaciones Coloquiales Val.Es.Co. -Briz y grupo Val.Es.Co. 2002; Corpus Val.Es.Co. 2.0.-Cabedo and Pons 2013-).

Key words: discourse markers, translation, colloquial Spanish, communicative approach, Val.Es.Co., DPDE.

\section{Introducción}

La enseñanza y el aprendizaje de los marcadores discursivos en la clase de E/LE presentan dificultades a docentes y alumnos: el docente debe emplear recursos y materiales que, siendo fieles a la corriente de estudios pragmático-discursivos 
publicados en las últimas décadas ${ }^{1}$, tengan un carácter didáctico que facilite su transmisión. A los alumnos, por su parte, les resulta más complicado aprender significados como el de los marcadores del discurso que trabajar con significados léxicos (conceptuales): los marcadores discursivos son unidades procedimentales y, por tanto, aquello que se aprende es la instrucción lingüística que codifican, asociada a su uso determinado (conectar, modalizar, interactuar) y a unas características contextuales concretas. Por esta razón, docentes y alumnos deben estar abiertos a métodos de estudio distintos, que vayan más allá de la mera memorización de listas y ejercicios tradicionales, y permitan comprender las propiedades lingüísticas de los marcadores discursivos (prosódicas, morfosintácticas, semánticas y, especialmente, pragmáticas).

Si a estas dificultades, inherentes al estudio de los marcadores discursivos, añadimos el hecho de trabajarlos en la clase de traducción, su tratamiento acaba convirtiéndose en un reto. En muchos casos, los estudiantes extranjeros de traducción no solo deben adquirir (o, al menos, consolidar) el significado de los marcadores discursivos de la lengua a la que más contenidos (textuales y audiovisuales) traducirán (lengua $\mathrm{B}$ e incluso $\mathrm{C}$ ), sino que, simultáneamente, deben aprender técnicas para su detección y adecuada traducción. Durante este proceso, las comparaciones entre lengua A (su lengua materna) y lengua(s) B (y/o C) serán constantes, y el profesor tendrá que evitar que el alumno acabe traduciendo literalmente los marcadores del discurso. En estos casos de traducción literal suele producirse una equiparación entre formas que da como resultado una interpretación errónea. Por ejemplo, una traducción formal del marcador español esto es suele ser id est en inglés -en contextos muy formales- o that is -en otros más coloquiales-. Sin embargo, esto no es siempre posible: el origen de numerosos marcadores discursivos varía notablemente entre lenguas y no hay un modo intuitivo de traducirlos, algo que ha sido demostrado sincrónica y diacrónicamente en diferentes trabajos contrastivos (Aijmer y Simon-Vandenbergen 2006): así sucede con la reformulación en inglés, frecuentemente expresada mediante $I$ mean, mientras el español se sirve de la forma o sea (Pons Bordería 2016: 292), elemento no marcado dentro del paradigma de marcadores de reformulación. Por estas razones, los aprendientes traductores necesitan ser capaces de manejar el significado procedimental de estos elementos yendo más allá de las puras formas.

Esta experiencia práctica parte de los diferentes problemas generados en este contexto específico de aprendizaje de español: el aula de traducción con español como lengua B. Proponemos un estudio piloto dirigido a estudiantes nativos de alemán (traducción alemán-español) pero que, dado su carácter contrastivo, puede aplicarse a otras combinaciones de lenguas, siempre que la lengua meta sea el español. Estos estudiantes no tienen un conocimiento del español completamente consolidado, sobre todo en lo referente a significados procedimentales como el de los marcadores discursivos (tanto en registros formales como informales). Para acotar nuestro objeto de estudio, esta experiencia se centra en aquellos marcadores discursivos propios de la conversación

1 Algunos de los trabajos teóricos más importantes publicados en el ámbito de los marcadores del discurso son, entre otros: Halliday and Hasan (1976); Zwicky (1985); Schourup (1999), Bazzanella (1986), Schiffrin (1985, 1987), Blakemore (1987, 2002), Fraser (1990, 1999, 2009), Schwenter (1996, 2000), Fischer (ed.) (2006); Fuentes Rodríguez (1987, 2009, 2012), Cortés Rodríguez (1991), Casado (1991, 1993), Briz Gómez (1993), Portolés (1993, 1998, 2010); Hansen (1998); Haselow (2011); Pons Bordería (1994, 1998, 2006, 2008); Martín Zorraquino (1999); Martín Zorraquino y Montolío Durán (1998); Briz et al. (2003); Cuenca (2006); Briz, Pons y Portolés (2008); Loureda y Acín (2010). 
oral coloquial a través de un conjunto de actividades que, si bien repararán en todas las propiedades de los marcadores discursivos arriba mencionadas, harán hincapié en los rasgos contextuales y los valores pragmáticos asociados a dichos marcadores. Estas propiedades son especialmente importantes no solo a la hora de aprenderlos, sino también cuando deban traducirlos.

\section{Marcadores del discurso, $E / L E$ y traducción: dificultades en su tratamiento}

El Diccionario de términos clave de E/LE, del Centro Virtual Cervantes (CVC), define los marcadores del discurso como "unidades lingüísticas invariables que señalan (marcan) la relación que se establece entre dos segmentos textuales, con ámbito supraoracional, que facilitan la cohesión textual y la interpretación de los enunciados". Esta definición es la que se maneja en los estudios canónicos publicados dentro del marco de la pragmática y el análisis del discurso (Schourup 1999; Schiffrin 1987; Fraser 1999), base de estudios posteriores. Una bibliografía que, a pesar de ser heterogénea en cuanto a la nomenclatura empleada (partículas discursivas, marcadores del discurso, conectores, etc.), presenta unanimidad a la hora de describirlos como unidades prosódicamente aisladas (por comas al escribirse o por la entonación al hablar); unidades morfológicamente invariables, pertenecientes a diferentes categorías gramaticales (desde interjecciones hasta adverbios, adjetivos o verbos); unidades sin integración sintáctica, autónomas, y combinables con otros elementos léxicos; y, por último, sin significado semántico conceptual codificado, sino procedimental (es decir, guían al oyente a la hora de procesar el sentido de los enunciados emitidos por el hablante).

Estos rasgos convierten a los marcadores discursivos en un objeto de estudio complejo: de hecho, su estudio en ámbitos de la lingüística aplicada (enseñanza de segundas lenguas, adquisición del lenguaje, traducción e interpretación, subtitulación, etc.) presenta dificultades a investigadores, traductores y docentes. Por ejemplo, en la clase de español como lengua extranjera (E/LE), el Plan Curricular del Instituto Cervantes (PCIC) y el Marco Común Europeo de Referencia para las lenguas (MCER) recomiendan su aprendizaje a partir de niveles elevados (al menos, C1).

Para esta labor, el docente puede utilizar los recursos incluidos en los diferentes manuales que oferta el mercado. Sin embargo, muchos de estos materiales no se centran por completo en el uso y funcionamiento pragmático-discursivo de los marcadores del discurso (y sí en otros rasgos, por ejemplo, morfosintácticos). Este hecho puede dificultar su aprendizaje en las aulas, incluso en niveles altos, puesto que aprender su significado simplemente memorizando listas o haciendo ejercicios sobre el papel que no tengan en cuenta su empleo real (sobre todo en el caso de los marcadores discursivos conversacionales) puede dar lugar a malos resultados.

Los alumnos necesitan adquirir una competencia comunicativa oral y escrita altas, relacionadas con los marcadores del discurso, y esta tarea se complica si al hecho de tener que estudiar significados procedimentales se le suma el uso de herramientas poco apropiadas. Se requieren, pues, materiales didácticos para la enseñanza de los marcadores discursivos en el aula de E/LE que, sobre todo, los traten explícitamente (La Rocca 2010: 3): un tratamiento implícito provoca que los alumnos no tomen conciencia de la importancia de su adquisición. Estos materiales también deberían reflejar una distinción clara entre oralidad y escritura: la mayoría de los materiales se centran en 
marcadores discursivos y conectores de lo escrito, y dejan el material oral, conversacional y coloquial en un segundo plano. Esto supone un error, puesto que el estudiante no acaba desarrollando todas las destrezas necesarias para adquirir el nivel deseado.

Por su parte, traducir los marcadores discursivos también se convierte en una tarea compleja y poco automática: es importante que en su traducción (sean cuales sean las lenguas de origen y meta) se tenga en cuenta cuál es su valor nuclear y cuáles son los valores pragmáticos ligados a la situación comunicativa en la que se utilizan (Borreguero 2011: 124). Las traducciones que solo se basan en la forma pueden llevar a un error traductológico que tiene como base una mala asimilación de los significados semántico y funcional subyacentes al marcador discursivo. En cambio, si el traductor detecta la instrucción de base en cada marcador discursivo, su traducción ganará en precisión y el proceso será más ágil.

La polifuncionalidad de los marcadores discursivos también es problemática: dificulta la búsqueda de un marcador equivalente en la lengua meta que se ajuste a cada una de las funciones que alberga el marcador de origen, problema ampliamente abordado en la bibliografía (Borreguero 2011: 129). No se recomienda, por ello, un enfoque únicamente semasiológico: aunque el traductor trabaje con una forma determinada, debe prestar atención al contexto y a la función o funciones que alberga, en un sentido onomasiológico, para mantener el sentido original en la lengua meta y encontrar una forma equivalente.

En suma, un escenario en el que el estudiante de español de nivel avanzado, que está consolidando sus conocimientos, sea también traductor, eleva el tratamiento de los marcadores discursivos al grado de complejidad más alto en la escala de aprendizaje. Queremos ofrecer una propuesta que, aunando ambos factores, ayude tanto a docentes como a alumnos. Detallamos esta propuesta en las próximas secciones.

\section{Tareas: objetivos, características, temporalización}

Como se ha señalado al inicio de este trabajo, el perfil de grupo al que se dirige este conjunto de actividades suele ser generalmente heterogéneo. Aunque es cierto que todos los alumnos tienen una edad igual o superior a los diecisiete años, y se encuentran en un contexto universitario (estudios de traducción), sus conocimientos de español pueden variar: hay alumnos que pueden haber alcanzado un nivel $\mathrm{B} 2$ o $\mathrm{C} 1 \mathrm{y}$ otros pueden situarse en niveles A2 o B1. Esta diferencia de niveles de aprendizaje supone la mayor de las dificultades añadidas al tratamiento de los marcadores discursivos en este contexto: el docente debe enfocar sus clases y seleccionar los contenidos para cada clase teniendo presente esta información. Su principal tarea es adaptarse a cada alumno y, a su vez, dar un tono general e integrador a cada sesión de aprendizaje.

En nuestro caso, los alumnos del grupo tenían un nivel de español medio-alto: eran capaces de escribir discursos elaborados $\mathrm{y}$, a excepción de algunas dificultades a nivel sintáctico y morfológico que se trabajaron a lo largo del curso, redactaban adecuadamente. En cuanto a la fluidez oral, era también media-alta (podría situarse entre un B2 y un $\mathrm{C} 1$ ): este era el punto adecuado para trabajar con marcadores discursivos, puesto que en niveles inferiores sería complicado seguir sin dificultades videos con fragmentos de conversación espontánea, tratar de describir contenidos en 
español, o explicar metalingüísticamente el funcionamiento de elementos procedimentales/funcionales como los marcadores discursivos.

La unidad didáctica, basada en un enfoque comunicativo, está compuesta por tres macro-tareas. Como el curso abarcaba también otros contenidos lingüísticos (cuestiones de morfología y sintaxis tradicional), era factible que estas ocuparan un total de sesenta minutos por sesión. El objetivo es que los alumnos vayan aprendiendo progresivamente y que conozcan nuevas herramientas de trabajo que les permitan, además de consolidar el significado de los marcadores discursivos en español, traducir sin dificultades del alemán al español. Las actividades propuestas son las siguientes:

\subsection{Su presencia es importante}

El profesor seleccionará dos programas de televisión/radio/grabaciones en los que exista un contexto de interacción natural (o seminatural), que impliquen una negociación comunicativa por parte de los hablantes y que, además, muestren un discurso sin planificación previa (o, al menos, no tan marcada). Uno será en alemán (el primero que visionarán o escucharán) y el otro en español. No hemos optado explícitamente por el género entrevista: queremos que los alumnos tomen conciencia de que los marcadores discursivos, junto con otros recursos conversacionales (pausas, entonación, léxico, alargamientos, etc.) ayudan al hablante a planificar su discurso sobre la marcha. Los programas mantienen en ocasiones el tono de entrevista, pero en contextos distintos que propician una interacción menos planificada.

Como este ejercicio sigue siendo de calentamiento, una toma de contacto, los fragmentos seleccionados serán cortos, y se les dará la transcripción para que puedan seguir los contenidos de ambos (especialmente para el fragmento en español). En el reverso de la hoja de transcripción encontrarán los mismos contenidos, pero sin los marcadores discursivos y/o partículas modales (para el alemán) que sí se hayan utilizado (el profesor los eliminará). De este modo, cuando vean las dos transcripciones (en especial, nuevamente, para el español), compararán y comprobarán la importancia de su uso para aportar al discurso diferentes valores pragmáticos. El profesor intentará, en la medida de lo posible, que se utilicen marcadores discursivos similares, que se presten a la traducción.

Los programas propuestos son Bares für Rares (para el alemán) y Planeta Calleja (para el español). El profesor puede seleccionar otros, siempre que no sean programas o series doblados de otro idioma al alemán o español: en estos casos, es posible que ciertas marcas modales o marcadores discursivos sean elección del traductor, y el alumno podría tener problemas si parte de este tipo de contenidos. Duración máxima del fragmento: 2 minutos. 

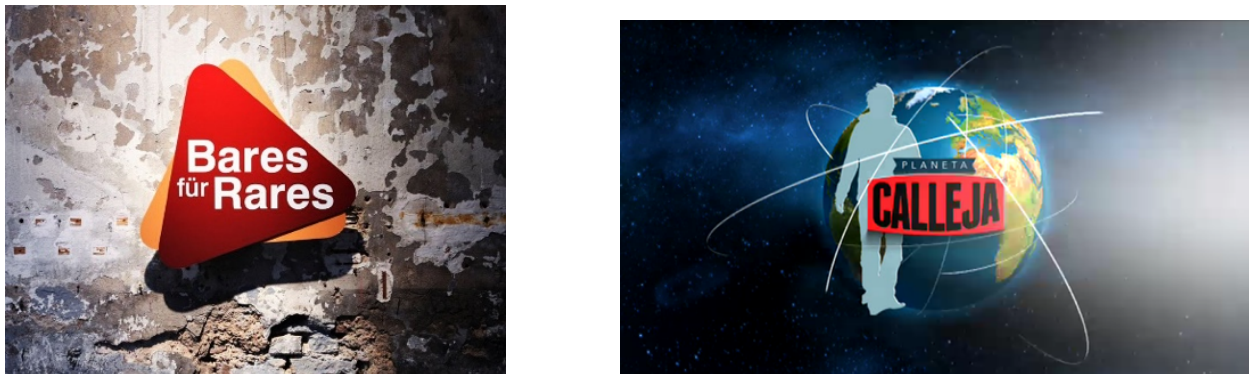

Figura 1: Fragmentos de programas seleccionados. Bares für Rares (alemán), Planeta Calleja (español).

\subsection{Todo depende del diccionario con el que se estudian}

En la siguiente sesión, los alumnos van a trabajar con nuevas herramientas, enfocadas ya al tratamiento de marcadores discursivos en español, y con el fin de que aprendan las correspondencias con el alemán desde un enfoque comunicativo y funcional. Una será la principal; la otra, de apoyo. Se busca consolidar el aprendizaje del significado de algunos marcadores discursivos tras la toma de contacto alemán-español llevada a cabo en sesiones previas.

La principal herramienta con la que trabajarán será el Diccionario de Partículas Discursivas del español ${ }^{2}$. No se les hará entrar en él de forma directa, sino inductiva: los alumnos trabajarán con transcripciones obtenidas del Corpus de Conversaciones Coloquiales del Grupo Val.Es.Co. (Briz y Grupo Val.Es.Co. 2002; Cabedo y Pons 2013): en concreto, de su versión en línea ${ }^{3}$. Los alumnos escucharán el fragmento conversacional en clase, transcripción mediante. La extensión de la transcripción corresponderá a unos dos o tres minutos de la grabación. Además, esta deberá contener suficientes marcadores discursivos para trabajar durante esa sesión. Seguidamente, intentarán detectar en la transcripción los diferentes marcadores discursivos utilizados: esta parte de la actividad se realizará entre todos, profesor y alumnos, para que resulte más sencillo, puesto que en las actividades de calentamiento han tomado contacto solo con unos pocos marcadores.

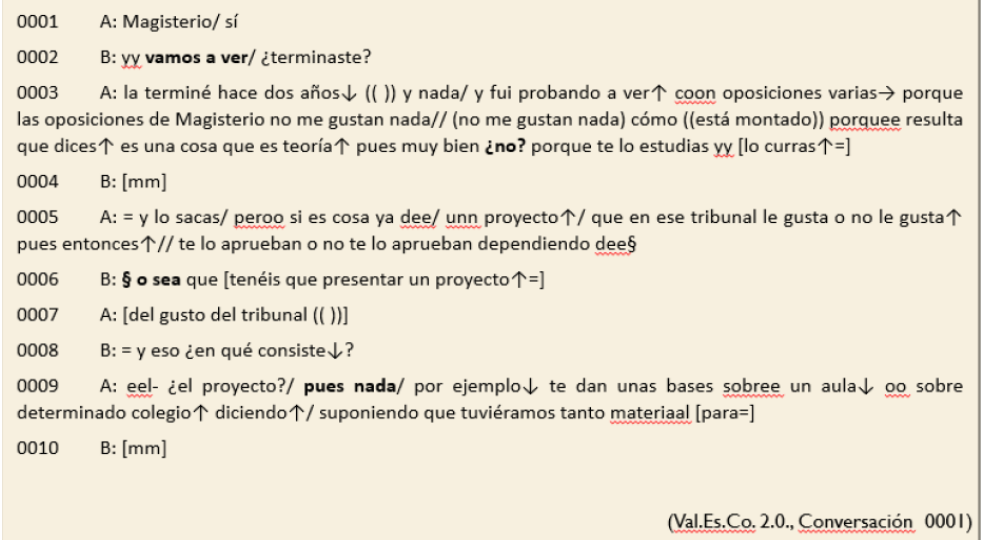

(Val.Es.Co. 2.0., Conversación 000I)

Figura 2. Extracto de conversación utilizado en la actividad. Corpus Val.Es. Co. 2.0.

\footnotetext{
2 (DPDE, cuya consulta está disponible en línea en: www.dpde.es)

${ }^{3}$ Corpus Val.Es.Co. 2.0., disponible en: http://www.valesco.es/?q=es/conversaciones).
} 
Tras encontrar los marcadores discursivos utilizados, el profesor pedirá a los alumnos que efectúen una búsqueda en un diccionario en línea que pueda definir los elementos destacados. Uno de ellos será el Diccionario de la Lengua Española (RAE, 2014). Tomando una muestra entre los distintos marcadores encontrados, los alumnos se percatarán de que el tratamiento de un marcador discursivo no es fácil ni tan solo para los propios nativos del idioma español. El marcador discursivo de reformulación o sea se define del siguiente modo:

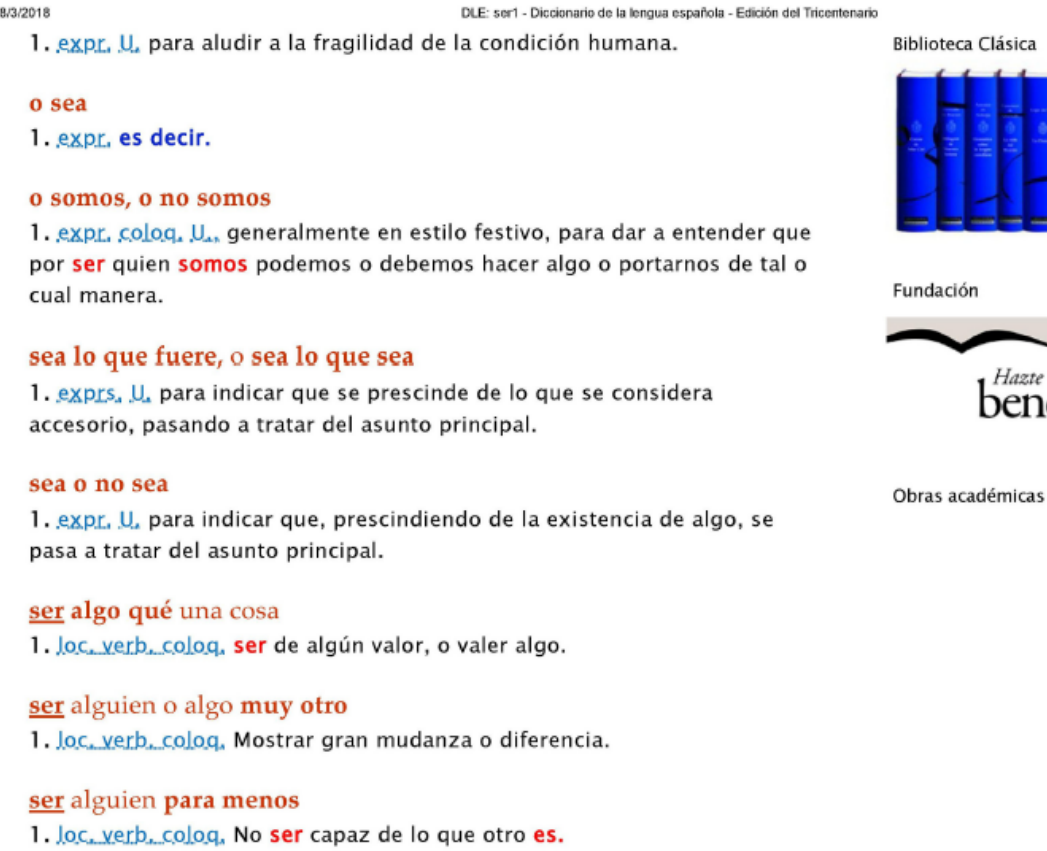

Figura 3. Definición de o sea en el Diccionario de la Lengua Española (RAE, 2014)

La única alusión explícita a su función de marcación discursiva se da en la definición 1. Esta definición dice que $o$ sea es una expresión equivalente a es decir, otro marcador discursivo de reformulación español que, además, no es completamente equivalente a $o$ sea. El marcador no tiene una definición propia; tampoco hay ejemplos que muestren su uso en contextos orales o escritos. El alumno, pues, tendrá dificultades para entender cómo funciona un elemento muy frecuente en contextos comunicativos reales.

Estas búsquedas harán que los alumnos reflexionen sobre la importancia de un correcto tratamiento de los marcadores discursivos para, por un lado, aprender bien su significado $\mathrm{y}$, por otro, traducirlos adecuadamente a otras lenguas.

Después del ejercicio de reflexión, empezarán a trabajar con el DPDE: los alumnos podrán ver la versatilidad de este recurso, que no solo recoge gran cantidad de marcadores discursivos (además, conversacionales) del español, sino también numerosos ejemplos (tomados de transcripciones, fragmentos literarios -para lo escrito-, entrevistas, etc.), audios en los ejemplos, diferentes contextos e incluso traducciones a otras lenguas. La disposición de contenidos en cada entrada del DPDE es bastante intuitiva y ordenada: hay un apartado que introduce más ejemplos, orales y escritos, que pueden servir al estudiante de apoyo formal y funcional para perfilar correctamente el funcionamiento del marcador discursivo buscado. También se aportan traducciones 
varias (al inglés, al italiano, al portugués), y estas traducciones siguen un mecanismo igual al que buscan las actividades aquí presentadas: basado en la instrucción procedimental codificada por los marcadores y sus respectivos contextos, y no simplemente en sus formas. La información prosódica y sobre puntuación también se aborda, siempre con varios ejemplos: los marcadores discursivos se separan de otros elementos que sí que forman parte del contenido proposicional de los enunciados gracias a sus rasgos prosódicos diferenciadores. Su posición también es tratada: los marcadores son muy versátiles, y por ello el alumno debe saber dónde es posible encontrarlos gracias a la exposición de diferentes contextos de uso reales. Por último, se incluye alguna información como su combinación con otros marcadores discursivos, la presencia del marcador buscado en fórmulas conversacionales asentadas en el español, e incluso datos sobre la posible variación geográfica de los marcadores discursivos, cubriendo así un amplio espectro descriptivo que ayudará al aprendiente y traductor.

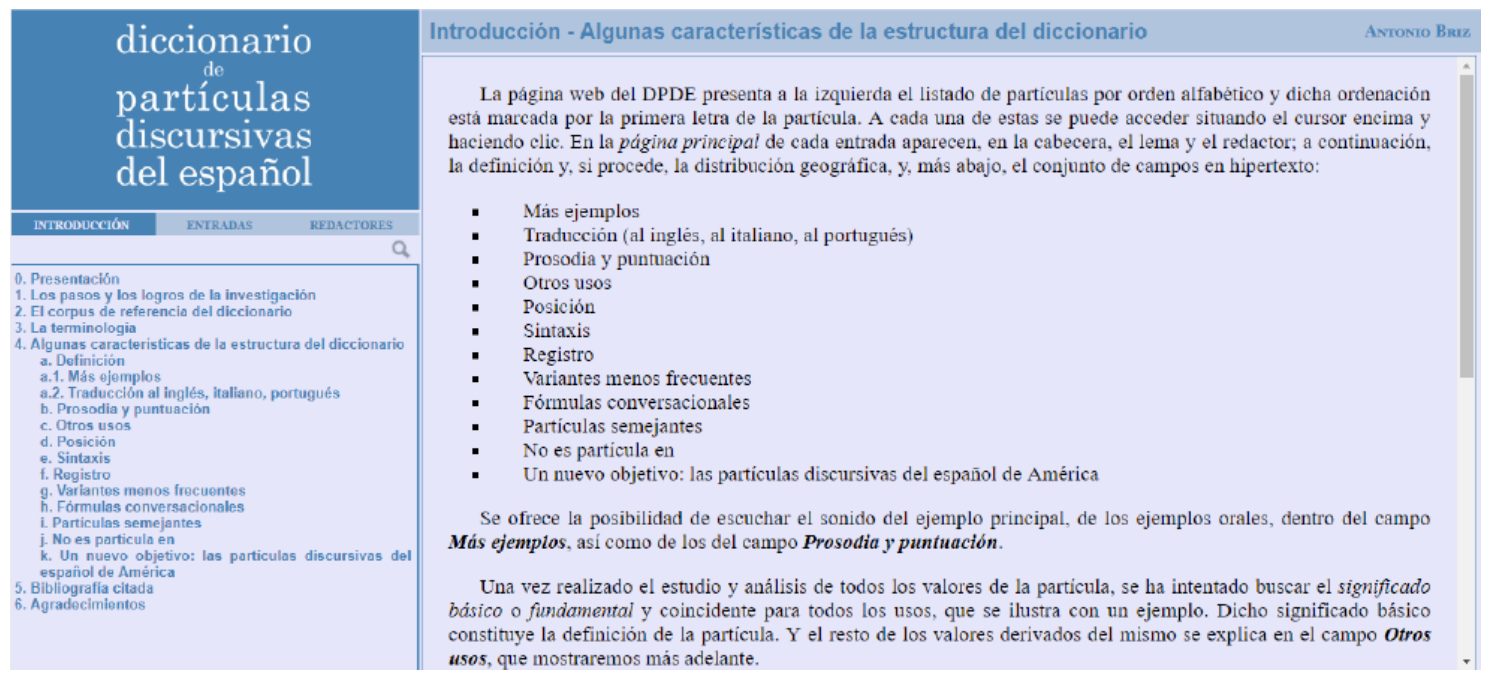

Figura 4. Diccionario de partículas discursivas del español (DPDE). Disposición de contenidos

Para acabar esta actividad, se organizará un debate entre los alumnos para que, en grupos, seleccionen cinco entradas del DPDE (cinco marcadores) e intenten, a partir de los contextos completos de los que disponen, buscar equivalentes en alemán. Este ejercicio permitirá evitar las traducciones literales, basadas únicamente en la forma del marcador.

\subsection{Aprendiendo y traduciendo}

En esta última sesión, los alumnos van a confeccionar su propio diccionario de partículas en alemán a pequeña escala. El objetivo es que aprendan a trabajar con estas unidades $\mathrm{y}$, sobre todo, con los contextos de uso en los que suelen emplearse. Nuevamente, en grupos de tres o en parejas, los alumnos seleccionarán un fragmento de un programa de televisión o radio. Si disponen de la transcripción, pueden utilizarla ya. Si no, la harán ellos mismos. Como ya han trabajado con transcripciones previamente, y han comprobado en qué situaciones el uso de modalpartikeln y marcadores discursivos es más frecuente, ya tendrán una base para seleccionar el contenido multimedia. Una vez seleccionado y trabajado el fragmento, se buscarán los marcadores discursivos y 
elementos modales empleados y se diseñarán entradas para la organización de la información (haciéndolo al igual que en el DPDE). Podrán apoyarse en el diccionario y, además, traducir al español cuando diseñen la pestaña "traducciones a otras lenguas". Lo expondrán al resto de compañeros para recibir comentarios y consejos de trabajo (a nivel de aprendientes y a nivel de traductores).

La disposición de los apartados del diccionario será similar a la que sigue el DPDE. Esta actividad permite a los alumnos comprobar hasta qué punto los significados procedimentales que están trabajando en las sesiones están asimilándose o no.

\begin{tabular}{|c|c|}
\hline Deutscher Diskurspartikeln Wörterbuch (DDW) & Definición \\
\hline $\begin{array}{l}\text { [Traducción del diccionario de partículas discursivas del } \\
\qquad \text { español] }\end{array}$ & Más ejemplos \\
\hline \multirow[t]{2}{*}{ Entradas seleccionadas: } & Traducción \\
\hline & Prosodia y puntuación \\
\hline Also & Otros usos \\
\hline Doch & Posición y sintaxis \\
\hline Mal & Registro \\
\hline \multirow[t]{2}{*}{ Ähm } & Variantes menos frecuentes \\
\hline & Formulas conversacionales \\
\hline Echt & No es partícula en \\
\hline
\end{tabular}

Imagen 2. Extracto de ficha DDW propuesta para los alumnos

Como puede observarse en la imagen, los alumnos deben completar información sobre las partículas modales del alemán que hayan escogido previamente. Para ello, buscarán más ejemplos, en contextos orales y escritos, podrán grabar sus propios ejemplos para tener muestras de audio que revelen información prosódica de las partículas trabajadas, delimitarán datos sobre el registro de uso y su sintaxis $\mathrm{y}$, sobre todo, buscarán equivalentes en español (directos o indirectos). Toda la información incluida deberá estar adecuadamente justificada, lo que les permite también adquirir conciencia de la importancia de las justificaciones en las labores traductoras.

Si los alumnos son capaces de llevar a cabo esta actividad sin muchas dificultades, será posible concluir que, como mínimo, ya comprenden cuál es la instrucción modal o discursiva básica de los marcadores discursivos, en alemán y español, con los que han trabajado.

\section{Conclusiones}

Este trabajo resume la propuesta piloto creada a partir de los resultados obtenidos en sesiones de trabajo con traductores alemán-español en la Universidad de Heidelberg (Alemania). Los objetivos de las tres actividades presentadas parten de las necesidades 
de aprendizaje de un grupo de trabajo especial compuesto por traductores que, además, están consolidando conocimientos de su lengua meta: el español. Este perfil de aprendiente no es tan común en el aula de E/LE, pero no significa que no exista: es muy habitual que en las universidades los estudiantes que trabajan con una segunda lengua presenten niveles de conocimientos sobre la misma muy dispares. Profesores y alumnos deben encontrar un punto medio: los alumnos de traducción alemán-español deben estar abiertos a trabajar en grupo y proporcionar input de conocimiento $(+1)$ a los alumnos que, quizá, tengan un nivel más bajo de español; los profesores, por su parte, deben atender a las necesidades comunicativas de todos los alumnos de su curso, sin centrarse solo en aquellos que tengan un nivel más avanzado, dejando atrás a los que no puedan seguir un ritmo tan fuerte, y sin centrarse solo en aquellos con un nivel más bajo, provocando que los alumnos que ya han adquirido unos conocimientos básicos repitan todos los contenidos ya vistos. Siendo los alumnos traductores, es importante que no se olvide el objetivo principal: ser capaces de traducir desde su lengua materna a la(s) lengua(s) meta, y traducir desde las otras lenguas a su lengua materna (traducción inversa). En la fase de aprendizaje en la que se encuentran (cerca de un $\mathrm{C} 1$, como mínimo un B2), los alumnos podrán consolidar significados procedimentales como los de los marcadores discursivos, que tan problemáticos son, y hacerlo mediante actividades orientadas hacia su campo de trabajo: la traducción. Pretendemos aplicar esta propuesta piloto en futuros cursos de enseñanza y traducción alemán-español.

\section{Bibliografía}

AIJMER, Karin y Simon-Vandenbergen, Anne-Marie (2006). «Pragmatic markers in contrast». Studies in Pragmatics, 2. Brill.

BORREGUERO, Margarita (2011). «La traducción de los marcadores del discurso: valores, funciones, posiciones y otros problemas». D. Sáez et al. (eds.); Últimas tendencias en traducción e interpretación (pp. 123-139). Franckfurt: Iberoamericana/Vervuert.

BRIZ, Antonio et al. (2002). Corpus de conversaciones coloquiales Val.Es. Co. Madrid: Arco

BRIz, Antonio, Salvador PONS y José PORTOLÉS (eds.) (2008). Diccionario de partículas discursivas del español. Consulta en línea en: www.dpde.es

CABEDO, Adrián y Salvador Pons (eds.). Corpus Val.Es.Co. 2.0. Consultado en línea en: http://www.valesco.es

Real Academia Española (2014). Diccionario de la Lengua Española. Consulta en línea en: www.rae.es

FRASER, Bruce (1999). «An approach to discourse markers». Journal of Pragmatics 14, 3, 383-398.

Instituto Cervantes (2018). Plan Curricular del Instituto Cervantes. Niveles de referencia del español. Madrid: Biblioteca Nueva.

LA RocCA, Marcella (2011). «Los marcadores del discurso en los manuales de Español/LE». RedELE: Revista electrónica de didáctica/Español Lengua Extranjera, 21. 
Pons, Salvador (2016). «Evolución diacrónica de o sea». BRAE, XCVI, CCCXIII, Enero-Junio de 2016.

ROSSARI, Corinne (1990). «Projet pour une typologie des opérations de reformulation». Cahiers de Linguistique Française 14, 151-171.

SCHOURUP, Lawrence (1999). «Discourse markers». Lingua, 107, 3-4, 227-265.

SCHIFFRIN, Deborah (1987). Discourse markers. Cambridge: Cambridge University Press / Washington DC: Georgetown University. 